\title{
PICC'ing Your Way into the Pericardium: A Potential Catastrophic Complication of a PICC Line
}

Young Im Lee ${ }^{1 *}$, Lina Miyakawa1, Michael Bergman ${ }^{2}$ and Robert L Smith ${ }^{3}$

${ }^{1}$ Icahn School of Medicine at Mount Sinai, Mount Sinai Beth Israel Hospital Center, New York, USA

${ }^{2}$ University of Virginia Health System, New York, USA

${ }^{3}$ NYU School of Medicine, VA Manhattan Harbor Medical Center, New York, USA

\begin{abstract}
This is a rare case of a peripherally inserted central catheter (PICC) that was found to be in the pericardiacophrenic vein on a post-procedure chest X-ray. An 82-year-old man was admitted to the medical ICU for severe sepsis with shock and a PICC was placed for vasopressor support. Malpositioning of a central venous catheter can lead to catastrophic results including, perforation, pericardial effusion and tamponade.
\end{abstract}

Keywords: Peripherally inserted central catheter; PICC; Pericardium; Central venous catheter

\section{Introduction}

There is an epidemic increase in use of central venous catheters. Malposition of these catheters can lead to catastrophic results [1]. Obtaining a post-procedure chest radiograph is common practice in an attempt to prevent these disasters. However with ultrasound guidance and EKG confirmation, more hospitals are moving away from obtaining post-procedure radiographs. This is a rare case demonstrating the importance of a confirmatory radiograph post-procedure.

\section{Case Report}

An 82-year-old man was admitted to the medical ICU for severe sepsis with shock due to Gram-negative pneumonia. Attempts to establish peripheral venous access were unsuccessful and thus a peripherally inserted central catheter (PICC) was placed using ultrasound guidance for prolonged antibiotic administration. A subsequent chest radiograph demonstrated the PICC traversing the left hemithorax and terminating in a position overlying the left ventricle. An arterial placement was initially suspected but venous placement was confirmed by measuring central venous pressure of $8 \mathrm{~cm} \mathrm{H}_{2} \mathrm{O}$ and $\mathrm{pO}_{2}$ of $32 \mathrm{mmHg}$ from the PICC line while simultaneous peripheral arterial $\mathrm{O}_{2}$ saturation on pulse oximetry was $97 \%$. A computed tomogram (CT) confirmed the PICC terminating in the pericardiacophrenic vein. The PICC was not used and removed without incident (Figures 1 and 2).

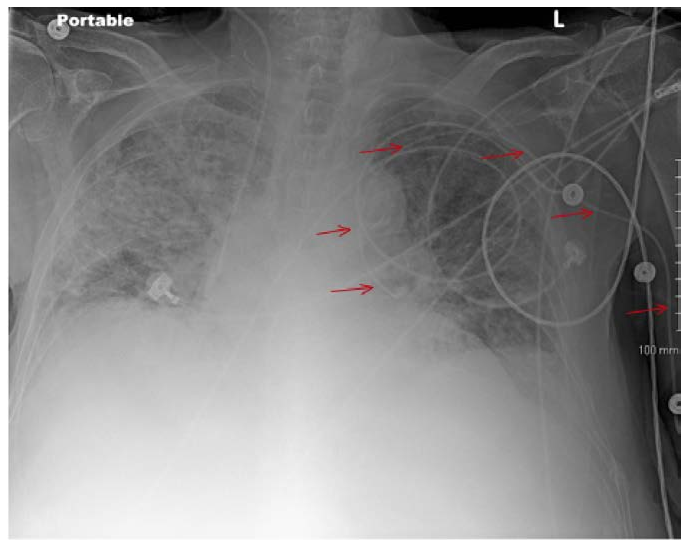

Figure 1: PICC line coursing through the left mediastinum with tip near the paracardiac space.

\section{Discussion}

Complications related to PICC lines can occur during insertion, while indwelling or after discontinuation. This case is a complication related to the insertion of the PICC into the pericardiacophrenic vein. The pericardiacophrenic vein traverses the mediastinum with its accompanying artery and phrenic nerve; it is a branch of the internal thoracic vein, draining into the brachiocephalic vein. Infusion of medications or fluids in a malpositioned central venous catheter may result in perforation and possible pericardial hemorrhage and tamponade [2]. These complications from malpositioned catheters are mostly described in literature relating to neonates. The incidence of this complication for adults is unknown. Although our patient developed no problems during a one-day cannulation, it is important to recognize the possibility of PICC

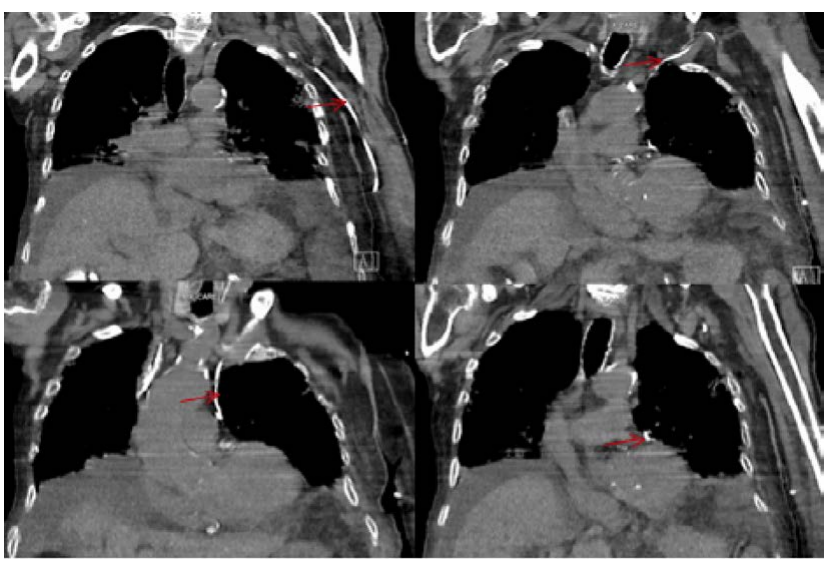

Figure 2: Computed tomogram demonstrating the PICC coursing through the central venous system with its tip in the pericardiacophrenic vein.

*Corresponding author: Young Im Lee, MD, Icahn School of Medicine, Mount Sinai Beth Israel, New York, USA, Tel: (212)420-2675; Fax: (212)420-4684 E-mail:Young.lee@mountsinai.org

Received August 16, 2017; Accepted August 25, 2017; Published August 29 2017

Citation: Lee YI, Miyakawa L, Bergman M, Smith RL (2017) PICC'ing Your Way into the Pericardium: A Potential Catastrophic Complication of a PICC Line. J Vasc Med Surg 5: 330. doi: 10.4172/2329-6925.1000330

Copyright: @ 2017 Lee YI, et al. This is an open-access article distributed under the terms of the Creative Commons Attribution License, which permits unrestricted use, distribution, and reproduction in any medium, provided the original author and source are credited. 
Citation: Lee YI, Miyakawa L, Bergman M, Smith RL (2017) PICC'ing Your Way into the Pericardium: A Potential Catastrophic Complication of a PICC Line. J Vasc Med Surg 5: 330. doi: 10.4172/2329-6925.1000330

Page 2 of 2

placement in the left paramediastinal space and its consequences even with ultrasound guidance $[3,4]$. This emphasizes the importance of radiography after insertion. A CT may be helpful to differentiate central venous line malposition, such as described in our case, from occasionally noted venous anomalies such as an aberrant left-sided superior vena cava. In the latter case, the central venous line may be used safely.

\section{References}

1. Zaman MH, Mitra P, Bondi E, Gintautas J, Abadir AR (1990) A rare malposition of the central venous catheter. Chest 98: 768-770.
2. Pettit J (2002) Assessment of infants with peripherally inserted central catheters: Part 1 . Detecting the most frequently occurring complications. Adv Neonatal Care 2: 304-315.

3. Shannon D (2014) Central venous catheter-induced pericardial effusion in a neonate: as case study and recommendations for practice. Neonatal Netw 33 : 341-348.

4. Ohki Y, Maruyama K, Harigaya A, Kohno M, Arakawa H (2013) Complications of peripherally inserted central venous cathter in Japanese neonatal intensive care units. Pediatr Int 55: 185-189. 